\title{
Requerimiento de paquetes eritrocitarios en neonatos: una revisión cualitativa
}

\section{Use of erythrocyte package in neonatology: a qualitative review}

\author{
Laura Isabel Jaramillo-Jaramillo, ${ }^{1}$ Juan Diego Villegas-Alzate, ${ }^{1}$ Camilo Ruiz-Mejía, ${ }^{1}$ Luis Felipe Álvarez-Hernández, ${ }^{1}$ \\ Lina María Martínez-Sánchez²
}

\begin{abstract}
Resumen
ANTECEDENTES: La trasfusión de hemoderivados, sobre todo de glóbulos rojos, es una de las intervenciones más comunes en la práctica médica diaria. En las unidades de neonatología la población objetivo de esta intervención suelen ser recién nacidos pretérmino, en particular los de alto riesgo con prematurez extrema y con bajo peso al nacer. OBJETIVO: Exponer los aspectos más relevantes de la indicación de hemoderivados a neonatos y la evidencia disponible al respecto: indicaciones, complicaciones, aspectos técnicos y medidas para reducir los riesgos asociados con el procedimiento, como las alícuotas de donante único.

DESCRIPCIÓN: Las transfusiones de productos sanguíneos a los neonatos son un procedimiento asociado con múltiples desenlaces desfavorables, como: enterocolitis necrosante, retinopatía del prematuro, hemorragia intraventricular, enfermedad pulmonar crónica, entre otros. La decisión de efectuar este procedimiento debe tomarse con base en las indicaciones establecidas y las posibles complicaciones.

CONCLUSIONES: El uso de alícuotas de donante único es uno de los nuevos desarrollos dirigido a disminuir los riesgos asociados y las complicaciones de incompatibilidad. PALABRAS CLAVE: Transfusión; productos sanguíneos; glóbulos rojos; enterocolitis;
\end{abstract} necrotizante; recién nacido, prematuro; enfermedades del recién nacido.

\section{Abstract}

BACKGROUND: Transfusion of blood products, especilly red blood cells, is one of the most common interventions in daily medical practice and in neonatal units the target population of this intervention is greater than that of other premature risks and with low birth weight.

OBJECTIVE: This review will be to generate the most important aspects of the use of erythrocyte package in the neonatal population and the available evidence in this regard. DESCRIPTION: Transfusions of blood products are multiple, especially in the neonatal population, among which include: necrotizing enterocolitis, retinopathy of prematurity, intraventricular hemorrhage, chronic lung disease, among others, so the decision to perform this procedure should be taken considering the indications and possible complications.

CONCLUSIONS: New protocols have been developed to minimize the risks associated with this procedure, among which the use of single-donor aliquots is highlighted, to reduce the complications of incompatibility.

KEY WORDS: Transfusion; Blood products; Red blood cells; Enterocolitis, Necrotizing; Infant, Premature; Infant, Newborn, Diseases.

\footnotetext{
${ }^{1}$ Estudiante en la Escuela de Ciencias de la Salud.

2 Especialista en Hematología, MSc, docente titular.

Universidad Pontificia Bolivariana, sede central Medellín, Colombia. Grupo de Investigación en Medicina Interna, Escuela de Ciencias de la Salud.

Recibido: 12 de diciembre 2017

Aceptado: 13 de agosto 2018

Correspondencia

Lina María Martínez Sánchez

linam.martinez@upb.edu.com

Este artículo debe citarse como Jaramillo-Jaramillo LI, Villegas-Alzate JD, Ruiz-Mejía C, Álvarez-Hernández LF, Martínez-Sánchez LM. Requerimiento de paquetes eritrocitarios en neonatos: una revisión cualitativa. Acta Pediatr Mex. 2018:39(5):298-306.
} 


\section{INTRODUCCIÓN}

La trasfusión de hemoderivados, sobre todo de paquete eritrocitario o de glóbulos rojos, es una de las intervenciones más comunes en la práctica médica diaria. En las unidades de neonatología la población objetivo de esta intervención son los recién nacidos pretérmino. ${ }^{1,2}$ En la mayoría de los casos el procedimiento se efectúa en un ambiente controlado, con la supervisión de especialistas y en unidades de cuidados intensivos o especiales neonatales. ${ }^{3,4}$ Sin embargo, a pesar de esto siguen corriéndose riesgos importantes y por ello suele recurrirse a pequeños volúmenes y solo se lleva a cabo cuando hay indicaciones específicas. ${ }^{5}$ Además, siguen desconociéndose las repercusiones clínicas de este procedimiento. ${ }^{5}$

Las trasfusiones a pacientes recién nacidos difieren, sustancialmente, de las que se efectúan a individuos de otros grupos etarios: mayor sensibilidad a los cambios de temperatura (termorregulación), mayor riesgo de hipoxia y cambios hematológicos propios del periodo neonatal. ${ }^{6}$ Además, las tasas de trasfusión se incrementan conforme disminuye la edad gestacional o el peso al nacer. Está reportado que de 50-94\% de los recién nacidos con muy bajo peso al nacer y hasta $95 \%$ de los recién nacidos con extremo bajo peso al nacer reciben, al menos, una trasfusión durante su estancia hospitalaria; esta población es más susceptible a los riesgos inherentes de este procedimiento. ${ }^{7}$

En pacientes pediátricos, e incluso en la población general, la tendencia actual es una actitud restrictiva a la indicación de hemoderivados debido a los riesgos que este procedimiento representa. Esta es la razón por la que se han intentado desarrollar nuevos protocolos, como el de alícuotas de donante único. Se intenta anticipar si el recién nacido requerirá más de una trasfusión y se reservan las unidades de un solo donante para obtener de allí los glóbulos rojos a trasfundir en las diferentes ocasiones. . $, 9,10^{2}$
Las trasfusiones de hemoderivados son, entonces, una medida terapéutica de gran utilidad en el tratamiento del paciente neonatal de alto riesgo pero que implica peligros importantes que todo el personal de salud que intervenga en la atención de estos pacientes debe conocer.

El objetivo de esta investigación fue: exponer los aspectos más relevantes de la indicación de hemoderivados a la población neonatal y la evidencia disponible al respecto: indicaciones, complicaciones, aspectos técnicos y medidas para reducir los riesgos asociados con el procedimiento, como las alícuotas de donante único.

\section{DESCRIPCIÓN}

Indicaciones en el recién nacido: relación riesgo-beneficio

La trasfusión de glóbulos rojos tiene como propósito primordial normalizar el aporte de oxígeno en todos los tejidos del cuerpo, aunque en algunos casos se utiliza con el propósito de mantener un adecuado volumen intravascular. ${ }^{11}$

Los recién nacidos, sobre todo los de prematurez extrema y con bajo peso al nacer, son receptores frecuentes de productos sanguíneos, más de la mitad de ellos con menos de 30 semanas de edad gestacional y más de $80 \%$ con peso al nacer menor de 1000 g requieren trasfusión de glóbulos rojos. Es necesario tener en cuenta que la decisión de efectuar este procedimiento en cualquier paciente (de cualquier edad) debe tomarse con cautela sopesando sus riesgos y beneficios porque se ha asociado con diferentes desenlaces desfavorables que incluyen: enterocolitis necrosante, hemorragia intraventricular, retinopatía del prematuro y enfermedad pulmonar crónica. De igual forma deben tenerse en cuenta complicaciones propias de cualquier trasfusión sanguínea como: trasmisión de infecciones (virales, bacterianas, parasitarias, fúngicas o por priones), 
enfermedad injerto-huésped, isoinmunización, trastornos hidroelectrolíticos agudos y errores en la administración, como la incompatibilidad de grupo. ${ }^{12-15}$ Además, la evidencia disponible referente a la indicación de esta intervención en este grupo de edad es limitada y ha comenzado a surgir recientemente, lo que dificulta aún más la decisión de llevar a cabo el procedimiento en un recién nacido. ${ }^{12}$

Por lo tanto, es decisivo conocer cuáles son las indicaciones actuales de la trasfusión de glóbulos rojos en recién nacidos y qué evidencia las respaldan, con el propósito de que el clínico pueda tomar una decisión sustentada para esta intervención. Enseguida se describen las principales indicaciones de trasfusión de glóbulos rojos en recién nacidos y la evidencia disponible al respecto.

Las indicaciones de la trasfusión de glóbulos rojos en recién nacidos son: ${ }^{10}$

- Choque hipovolémico con pérdida sanguínea aguda (hemorrágico).

- Pacientes con requerimientos de $\mathrm{FiO}_{2}$ mayores de $35 \%$ o soporte ventilatorio no invasivo con presión positiva continua o invasiva con una presión media de 6-8 $\mathrm{cm}$ de agua.

\section{Anemia:}

- Hematocrito entre $30-35 \%$ o hemoglobina entre 10-12 g/dL en el contexto de un paciente críticamente enfermo y que se considera que la trasfusión de glóbulos rojos mejorará la entrega de oxígeno a los órganos vitales.

- Recién nacidos con hematocrito entre 20$30 \%$ o hemoglobina entre $6-10 \mathrm{mg} / \mathrm{dL}$, si el recién nacido se encuentra críticamente enfermo o tiene ventilación mecánica asistida.

- Hematocrito menor de 30\% y alguna de las siguientes condiciones:
- Administración de oxígeno suplementario.

- Ventilación con presión positiva continua o ventilación mecánica menor de $6 \mathrm{~cm}$ de agua.

- Episodios significativos de apnea y bradicardia definidos como: más de 9 episodios en 12 horas o 2 episodios en 24 horas que requieren administración de presión positiva con máscara, con dosis adecuadas de metilxantinas.

- Frecuencia cardiaca mayor de 180 latidos por minuto o respiratoria mayor a 80 respiraciones por minuto por más de 24 horas.

- Deficiente ganancia de peso (menos de 10 g en un lapso mayor a 4 días con aporte calórico mayor de 100 kcal/kg/día).

- Inmediata intervención quirúrgica.

- Hematocrito menor de $20 \%$ en pacientes asintomáticos si tiene un recuento de reticulocitos menor de 100 x 103/mcL.

Hay algunas circunstancias en las que debe evitarse la trasfusión de glóbulos rojos: ${ }^{10}$

- No está indicada solo por un volumen de extracción elevado.

- No transfundir únicamente por concentraciones de hematocrito aisladamente bajas.

Evidencia disponible acerca de las principales indicaciones de trasfusión de glóbulos rojos a recién nacidos (anemia y choque hemorrágico)

\section{Choque hemorrágico}

La mayor parte de los choques hemorrágicos en el recién nacido ocurren al momento de nacer y son originados, principalmente, por: hemorragias placentarias, síndrome de trasfusión feto-fetal, hemorragia materno-fetal, inserción velamentosa del cordón, o ruptura de éste; las causas traumáticas de sangrado son poco comunes. ${ }^{13}$ 
El tratamiento general sugerido para estos casos incluye la administración de bolos de solución salina isotónica en volúmenes de $10-20 \mathrm{~mL} /$ $\mathrm{kg}$, mientras están disponibles las unidades de glóbulos rojos que se administran en volúmenes similares. Este enfoque no ha sido evaluado en ensayos clínicos y se basa principalmente en opiniones de expertos. ${ }^{13,16}$

\section{Anemia}

La anemia neonatal (concentración de hemoglobina o hematocrito inferior a -2 desviaciones estándar del promedio esperado para la edad postnatal) representa una problemática frecuente en las unidades de cuidado intensivo neonatal. ${ }^{16}$ A últimas fechas, las bajas concentraciones de hemoglobina se han asociado con mortalidad en neonatos pretérmino de menos de 32 semanas de edad gestacional. Además, la anemia por largos periodos puede afectar el crecimiento cerebral de los recién nacidos pretérmino y a término; de ahí la importancia de la interpretación de los parámetros hematológicos indicativos de anemia y de la repercusión de la terapia transfusional en su tratamiento. Las indicaciones de trasfusión son claras en caso de anemia por choque hemorrágico; sin embargo, en otros casos la decisión de trasfundir o no debe basarse en la integración de parámetros clínicos, de laboratorio y las necesidades fisiológicas y el estado de salud general del recién nacido. ${ }^{16,17}$

El principal problema de la trasfusión de glóbulos rojos a recién nacidos reside en el umbral de hemoglobina y hematocrito que indica la necesidad de iniciar la trasfusión. ${ }^{16}$ En un estudio efectuado por Bell EF y su grupo se comparó el uso de umbrales "liberales" vs "restrictivos" en recién nacidos pretérmino y se encontró que ambos enfoques fueron bien tolerados, sin encontrar diferencia en el número de recién nacidos que evitaron nuevas transfusiones y reportaron mayor frecuencia de desenlaces neurológicos adversos (hemorragia intraparenquimatosa, leucomalacia periventricular) en el grupo de terapia restrictiva. ${ }^{18}$ De igual forma, en una revisión sistemática realizada por Whyte $\mathrm{R}$ no se encontraron diferencias estadísticamente significativas entre límites altos o bajos de hemoglobina para iniciar la trasfusión de glóbulos rojos. ${ }^{13}$

En conclusión, el inicio o no de trasfusión de glóbulos rojos en pacientes con anemia depende del criterio del clínico y de su interpretación del estado del paciente con base en parámetros hematológicos y el estado general de salud del paciente.

\section{Aspectos técnicos del procedimiento en recién nacidos}

Los recién nacidos son receptores de componentes sanguíneos particularmente vulnerables, por lo que se deben aplicar medidas de seguridad especiales debido a su tamaño e inmadurez en el desarrollo, buscando la mayor esperanza de vida posible. ${ }^{19,20}$

Los bancos de sangre deben evitar la exposición de los receptores a múltiples donantes utilizando los paquetes de intercambio neonatal o pedi paks porque cada uno tiene entre 4 y 5 alícuotas del mismo donante. ${ }^{20}$

En el Cuadro 1 se mencionan las dosis a trasfundir de componentes sanguíneos en niños..$^{21,22}$

\section{Cuidados especiales}

Se recomienda que cuando se va a llevar a cabo una trasfusión masiva en el recién nacido se ajuste la temperatura de la sangre para evitar una baja de 0.7 a $2.5{ }^{\circ} \mathrm{C}$ en el recién nacido. En unidades de fototerapia también hay que tener precauciones porque la sangre puede hemolizarse dependiendo de la ubicación del equipo de trasfusión. ${ }^{23}$ Las plaquetas no pueden calentarse porque se altera su función. ${ }^{21}$ 
Cuadro 1. Transfusión de componentes sanguíneos en niños ${ }^{21,22}$

\begin{tabular}{|c|c|c|c|}
\hline Hemocomponente & Objetivo & Dosis & Resultado esperado \\
\hline $\begin{array}{l}\text { Concentrado de } \\
\text { glóbulos rojos }\end{array}$ & $\begin{array}{l}\text { Aumentar la capacidad de } \\
\text { transporte de oxigeno }\end{array}$ & $10-15 \mathrm{ml} / \mathrm{kg}$ & $\begin{array}{l}\text { Aumenta } 2 \text { a } 3 \mathrm{~g} / \mathrm{dL} \text { de } \\
\text { hemoglobina }\end{array}$ \\
\hline Concentrado de plaquetas & $\begin{array}{l}\text { Prevenir sangrado por alteración } \\
\text { cuantitativa o } \\
\text { cualitativa de plaquetas }\end{array}$ & $5-10 \mathrm{ml} / \mathrm{kg}$ & Aumenta 50,000 plaquetas $/ \mathrm{mm}^{3}$ \\
\hline Plasma fresco congelado & Reponer factores de coagulación & $10-15 \mathrm{ml} / \mathrm{kg}$ & $\begin{array}{l}\text { Aumento de } 15 \text { a } 20 \% \text { de los } \\
\text { factores }\end{array}$ \\
\hline Crioprecipitado & $\begin{array}{l}\text { Tratamiento y profilaxis en } \\
\text { deficiencia de factores }\end{array}$ & $\begin{array}{c}1-2 \mathrm{U} / 10 \mathrm{~kg} \mathrm{de} \\
\text { peso }\end{array}$ & $\begin{array}{l}\text { Aumento de } 60 \text { a } 100 \mathrm{mg} / \mathrm{dL} \text { de } \\
\text { fibrinógeno }\end{array}$ \\
\hline
\end{tabular}

El suero fisiológico acompañado de adenina, glucosa y manitol, que incluye el concentrado de eritrocitos, representa un riesgo por su alta toxicidad renal y puede causar alteración de la volemia y flujo cerebral del recién nacido, pero la trasfusión de pequeños volúmenes (5 a 15 mL/ $\mathrm{kg}$ ) de concentrado de eritrocitos con glucosa y manitol son seguras. ${ }^{21}$

\section{Leucorreducción}

Los leucocitos que se encuentran en los componentes sanguíneos que van a ser transfundidos pueden generar reacciones inmunológicas: reacción febril no hemolítica, enfermedad de injerto contra huésped e inmunosupresión por efectos inmunomoduladores, para evitarlas es recomendable el uso de filtros leucocitarios. ${ }^{21}$

\section{Irradiación}

La irradiación de componentes sanguíneos está indicada para evitar la proliferación de linfocitos del donante en el receptor y evadir que se genere una reacción postransfusional. La irradiación disminuye la supervivencia de los glóbulos rojos y aumenta el potasio extracelular, por esto el uso del componente debe tener irradiación reciente para limitar los efectos del potasio libre. ${ }^{21}$

\section{Lavado con solución salina}

El objetivo es disminuir las proteínas plasmáticas que pueden desencadenar una reacción adversa en el receptor. Las indicaciones son muy específicas: pacientes con reacción anafiláctica por déficit de lgA y reacción alérgica en transfusiones previas y recién nacidos con trombocitopenia aloinmune. $^{21}$

\section{Reducción del sobrenadante}

En el estudio efectuado por Serrano y sus colaboradores se demostró que, al reducir el sobrenadante por centrifugación, se facilita el uso de glóbulos rojos, además de aumentar su concentración en las alícuotas centrifugadas en $40 \pm 7 \%$, lo que puede tener un efecto adicional al disminuir la coexistencia de sustancias transfundidas al neonato. ${ }^{24}$

\section{Transfusiones de glóbulos rojos frescos o almacenados}

Durante años se han administrado glóbulos rojos frescos con más menos siete días o unidades de máximo 35 a 42 días de almacenamiento. Ambas opciones implican riesgos para los neonatos; sin embargo, las unidades almacenadas de un mismo donante acumulan sustancias tóxicas como: 
potasio, amoniaco, baja capacidad de descarga de oxígeno por la pérdida de 2,3 difosfoglicerato; a pesar de ello, estas desventajas no superan los riesgos de la exposición alogénica de diferentes donantes. ${ }^{25,26}$

\section{Complicaciones de las transfusiones}

La población neonatal es especialmente susceptible a las complicaciones derivadas de la transfusión de hemoderivados. Algunos estudios sugieren que la trasfusión de glóbulos rojos incrementa la mortalidad neonatal, sobre todo en recién nacidos con extremo bajo peso al nacer. ${ }^{7}$

Entre las principales complicaciones asociadas con la transfusión de glóbulos rojos en recién nacidos destacan: ${ }^{7,9}$

- Enterocolitis necrosante

- Hemorragia intraventricular

- Retinopatía del prematuro

- Enfermedad pulmonar crónica

- Sobrecarga de volumen

- Hemólisis

- Sensibilización a antígenos eritrocitarios

- Hiperpotasemia

- Reacción injerto-contra-huésped

- Infecciones

Las complicaciones pulmonares están claramente establecidas en los pacientes adultos e igualmente se han estudiado en población neonatal. Su fisiopatología se atribuye a la lesión producida por el hierro y los radicales libres. El daño pulmonar se ha reportado, incluso, 29 veces mayor en recién nacidos con muy bajo peso al nacer cuando reciben más de $30 \mathrm{~mL}$ de hemoderivados en el periodo neonatal. En los diferentes estudios no se han obtenido resultados estadísticamente significativos, por lo que esta afirmación es aún motivo de controversia. ${ }^{7}$ De igual forma, por la existencia de evidencia contradictoria, el riesgo de enterocolitis necrosante asociada con la transfusión de glóbulos rojos es aún motivo de controversia. ${ }^{4,27}$

\section{Un nuevo panorama: donante único}

Este término abarca no sólo a los voluntarios que donan parte de su contenido hemático y que, posteriormente, se almacena o procesa de acuerdo con las necesidades, sino que también incluye a la medicina transfusional, que consta de la inmunoterapia, la terapia celular y de tejidos. ${ }^{28,29}$ Se entiende por donante único a la transfusión alogénica de las células sanguíneas provenientes de un solo individuo, con lo que disminuye el riesgo de complicaciones debido a situaciones de incompatibilidad..$^{30}$ En un estudio efectuado por Strauss y su grupo ${ }^{30}$ se consiguió determinar, en una población pediátrica, que la modalidad de donante único debe considerarse en pacientes con cirugía electiva y con necesidad anticipada de glóbulos rojos. ${ }^{31}$ Los recién nacidos pretérmino requieren múltiples transfusiones de volúmenes pequeños de glóbulos rojos, circunstancia que los deja expuestos a un elevado número de donantes, y al incremento del riesgo de complicaciones por incompatibilidad. El protocolo de donante único surge como una alternativa para minimizar estos riesgos; para esto, se recolecta la sangre de un donante y se distribuye en alícuotas individuales con las condiciones de calidad requeridas y con máximo de almacenamiento de 42 días, para que los riesgos de filtración del potasio intracelular, hemoglobina, lactato deshidrogenasa (LDH) y la posible contaminación bacteriana, no sobrevengan en este periodo. ${ }^{32,33}$

Este protocolo de tratamiento es, sobre todo, útil en pacientes que requieren transfusión de concentrados de plaquetas porque el estándar inicial de atención en salud en pacientes con hemorragia aguda secundaria a trombocitopenia 
consistía en la transfusión de concentrados de múltiples donantes. Las tasas de isoinmunización con ese protocolo son elevadas y se requirieren plaquetas de donantes con tipos específicos de HLA. ${ }^{30,34}$ Esta necesidad médica impulsó el desarrollo de procedimientos de aféresis que permiten la recolección de una dosis alta de plaquetas HLA-compatibles de un solo donante, denominada plaquetas de donante único, ${ }^{30}$ dando inicio al protocolo de donante único con otros hemoderivados. ${ }^{35}$

Uno de los principales problemas de la transfusión tradicional, en especial en los pacientes con alteraciones hematológicas, es la resistencia plaquetaria, que en algunos casos puede sobrevenir como una reacción febril característica. ${ }^{34,36}$ En la mayoría de los casos este rechazo es dado por fenómenos inmunitarios (que se incrementan cuando hay exposición a múltiples donantes), razón por la que la disponibilidad de plaquetas, específicamente HLA-compatibles, puede ser decisiva. ${ }^{30}$ Se recomienda el uso de plaquetas por aféresis de donante único que carece de contaminación de eritrocitos y evita la necesidad de inmunoprofilaxis en esta población. ${ }^{37}$

Otro de los riesgos más comunes de la transfusión convencional es la reacción séptica a la transfusión plaquetaria (SPTR por sus siglas en inglés: Septic platelet transfusion reaction). ${ }^{38,39,40}$ En Estados Unidos está reportado que esta reacción se presenta en 1 de cada 4200 transfusiones, razón por la que surgen nuevos métodos de transfusión para reducir el riesgo, como el mecanismo de donante único que, según el estudio de Ness y colaboradores, logró reducir la reacción séptica a la transfusión plaquetaria a 1 caso por cada 15,098 trasfusiones. . $^{39,40}$

Una ventaja más es que el método de donante único, en contraste con los métodos de recolección de sangre completa, que requieren la agrupación de plaquetas de cuatro a seis do- nantes para conseguir una dosis terapéutica de plaquetas (> $3 \times 10^{11}$ plaquetas), proporciona una dosis terapéutica completa por sí sola. ${ }^{41}$ Asimismo, los estudios han demostrado una relación directa entre la cantidad de productos sanguíneos que reciben los pacientes y la aparición de complicaciones graves infecciosas o no infecciosas. La utilización de aféresis por donante único reduce la necesidad de múltiples transfusiones y se traduce en menos complicaciones. Además, este protocolo reduce los costos de manipulación porque elimina la necesidad de agrupación de donaciones y evita la repetición de algunas pruebas infecciosas. ${ }^{42-46}$

\section{CONCLUSIÓN}

Las transfusiones de hemoderivados son medidas terapéuticas de gran utilidad en el tratamiento de recién nacidos de alto riesgo; pero también son procedimientos con riesgos importantes, sobre todo en el recién nacido pretérmino gravemente enfermo. La decisión de llevar a cabo esta intervención debe tomarse con cautela teniendo en cuenta las indicaciones y los riesgos asociados con el procedimiento. Asimismo, debe garantizarse que las condiciones en las que se efectúa el procedimiento sean las apropiadas para minimizar al máximo el riesgo de complicaciones. El protocolo de donante único surge, entonces, como una nueva alternativa para incrementar los índices de seguridad y reducir las complicaciones por incompatibilidad asociadas con la transfusión de productos sanguíneos. En la actualidad, este protocolo se aplica, sobre todo, para la transfusión de concentrados de plaquetas; es importante explorar en futuros estudios su utilidad en la transfusión de otros hemoderivados.

\section{REFERENCIAS}

1. Banerjee J, et al. Blood transfusion in preterm infants improves intestinal tissue oxygenation without alteration in blood flow. Vox Sang. 2016;111(4):1-10. https://doi. org/10.1111/vox.12436 
Jaramillo-Jaramillo L I et al. Paquetes eritrocitarios en neonatos

2. Heddle NM, et al. Effect of Short-Term vs. Long-Term Blood Storage on Mortality after Transfusion. N Eng J Med. 2016;375(20):1937-45. DOI: 10.1056/NEJMoa1609014

3. Finn D, Dorrian A, et al. Emergency uncross-matched blood transfusions in a tertiary neonatal unit. Acta Paed. 2016; 106:218-22. https://doi.org/10.1111/apa.13646

4. Miller HD, et al. Assessment of Mesenteric Tissue Saturation, Oxygen Saturation, and Heart Rate Pre- and PostBlood Transfusion in Very Low-Birth-Weight Infants Using Abdominal Site Near-Infrared Spectroscopy. Adv Neonatal Care. 2017;17(5):E3-E9. https://doi.org/10.1097/ ANC. 0000000000000417

5. Ministerio de Salud y Protección Social. Guía de práctica clínica basada en evidencia para el uso de componentes sanguíneos/hemocomponentes. Bogotá; 2017. Módulo 10. P 395-404.

6. Oliveira E, Herdy V, Pereira D, Dallabela F, Vidal J, Linhares V. Nurses' knowledge of blood transfusion in neonate. Rev Gaúcha Enferm. 2017;38(1):e63557. ttp://dx.doi. org/10.1590/1983-1447.2017.01.63557

7. OJO. NO SE ENCONTRÓ Wang YC, Chan OW, Chou M, Hong $\mathrm{P}$, Chu SM, Hsu JF, et al. Red Blood Cell Transfusion and Clinical Outcomes in Extremely Low Birth Weight Preterm Infants. Pediatr Neonatol. 2017; 58: 216-22.

8. Saldaña-Casas O, De la Torre-Fernández A, Guzmán-García M, Blas-Mendiola C, Gómez-Gómez M, Saltigeral-Simental P. Transfusión de componentes sanguíneos en un hospital privado para niños. Rev Mex Pediatr. 2005; 72(2); 65-9.

9. Arca G, Carbonell-Estrany X. López J, Figueras J, Fernández B, Blanco D, Closa R, Narbona E, et al. Anemia neonatal. En: Junta Directiva de la Sociedad Española de Neonatología (SEN). Protocolos de Neonatología. 2. ed. España: Asociación Española de Pediatría (AEP). 2003. p: 362-71.

10. Restrepo N. Manejo de componentes sanguíneos en recién nacidos. Programa de educación continua en pediatría. Sociedad Colombiana de Pediatría. 2013;12(2):59-69.

11. Parker RI. Transfusion in Critically III Children: Indications, Risks, and Challenges. Crit Care Med. 2014; 42:675-90. doi: 10.1097/CCM.0000000000000176

12. Kelly AM, Williamson LM. Neonatal transfusion. Early Hum Dev. 2013;89(11):855-60. doi: 10.1016/j.earlhumdev.2013.08.025.

13. Whyte RK, Jefferies AL; Canadian Paediatric Society, Fetus and Newborn Committee. Red blood cell transfusion in newborn infants. Paediatr Child Health. 2014;19(4):213-22.

14. Keir A, Pal S, Trivella M, Lieberman L, Callum J, Shehata N, et al. Adverse effects of red blood cell transfusions in neonates: a systematic review and meta-analysis. Transfusion. 2016;56(11):2773-80. doi:10.1111/trf.13785.

15. Folarte I, et al. Transfusiones de glóbulos rojos en recién nacidos de muy bajo peso de nacimiento. Rev Chil Pediatr 2014; 85 (3): 298-303. http://dx.doi.org/10.4067/S037041062014000300005
16. Colombatti R, Sainati L, Trevisanuto D. Anemia and transfusion in the neonate. Semin Fetal Neonatal Med. 2016;21(1):2-9. doi: 10.1016/j.siny.2015.12.001.

17. Banerjee J, et al. Haemoglobin level at birth is associated with short term outcomes and mortality in preterm infants. BMC Med. 2015;13:16. https://doi.org/10.1186/ s12916-014-0247-6

18. Bell EF, Strauss RG, Widness JA, Mahoney LT, Mock DM, Seward VJ, et al. Randomized trial of liberal versus restrictive guidelines for red blood cell transfusion in preterm infants. Pediatrics. 2005;115(6):1685-91.

19. Mangal R, Meyer EK, Widness JA. Research Opportunities to Improve Neonatal Red Blood Cell Transfusion. Transfus Med Rev 2016;30: 165-73. https://doi.org/10.1016/j. tmrv.2016.06.005

20. New HV, et al. Guidelines on transfusion for fetuses, neonates and older children. Br J Haematol. 2016;175(5):784828. doi: 10.1111/bjh.14233.

21. Rodrigues L, Barbosa A, Barbosa C, Basílio J, Novello $\mathrm{R}$, Pessôa $\mathrm{V}$, et al. Transfusión de hemocomponentes en niños: ¿qué, cuándo y cómo usar?. Resid. Pediatr. 2015;5(1):14-20.

22. Comité médico asesor del BloodCenter. Guias de transfusión pediátrica. Wisconsin. Wisconsin BloodCenter; 2015.

23. Pozo A. Transfusión en neonatología. Rev. Hosp. Mat. Inf. Ramón Sardá. 2009;28(2):86-96

24. Serrano K, Pambrun C, Levin E, Devine DV. Supernatant reduction of stored gamma-irradiated red blood cells minimizes potentially harmful substances present in transfusion aliquots for neonates. Transfusion. 2017;57:3009-18. doi:10.1111/trf.14270.

25. Strauss RG. 2008 Emily Cooley Memorial Lecture: lessons learned from pediatric transfusion medicine clinical trials.. . a little child shall lead them. Transfusion. 2009;49(9):19962004. doi: 10.1111/j.1537-2995.2009.02267.x.

26. Strauss RG. How I transfuse red blood cells and platelets to infants with the anemia and thrombocytopenia of prematurity. Transfusion. 2008;48(2):209-17. doi: 10.1111/j.1537-2995.2007.01592.x.

27. Mohamed A, Shah PS. Transfusion Associated Necrotizing Enterocolitis: A Meta-analysis of Observational Data. Pediatrics. 2012;219(3):529-763.

28. Contreras $D$, Martínez M. Medicina transfusional en el siglo XXI. Rev. Med. Clin. CONDES, 2015; 26(6) 726-743

29. Kirkley SA, Blumberg N. Use of single donor platelets. Blood Rev. 1994 Sep;8(3):142-7. Review.

30. Ness PM, Campbell-Lee SA. Single donor versus pooled random donor platelet concentrates. Curr Opin Hematol. 2001;8(6):392-6.

31. Strauss RG, Wieland MR, Randels MJ, Koerner TA. Feasibility and success of a single-donor red cell program for pediatric elective surgery patients. Transfusion. 1992;32(8):747-9. 
32. Strauss RG, Villhauer PJ, Cordle DG. A method to collect, store and issue multiple aliquots of packed red blood cells for neonatal transfusions. Vox Sang. 1995;68(2):77-81.

33. Strauss RG. Controversies in the management of the anemia of prematurity using single-donor red blood cell transfusions and/or recombinant human erythropoietin. Transfus Med Rev. 2006;20(1):34-44. https://doi. org/10.1016/j.tmrv.2005.08.003

34. Neth R, Gallo R, Greaves M, Janka G, editors. Modern trends in human leukemia VI: new results in clinical and biological research including pediatric oncology. Wilsede Joint Meeting on Pediatric Oncology 3, Hamburg, June 21/22, 1984. Berlin: Springer; 1985.

35. Böck $M$, Glaser A, Pfosser A, Schleuning $M$, Heim MU, Mempel W. Storage of single-donor platelet concentrates: metabolic and functional changes. Transfusion. 1993;33(4):311-5

36. Chambers LA, Kruskall MS, Pacini DG, Donovan LM. Febrile reactions after platelet transfusion: the effect of single versus multiple donors. Transfusion. 1990;30(3):219-21.

37. Molnar R, et al. Absence of $D$ alloimmunization in $D$ - pediatric oncology patients receiving $D$-incompatible singledonor platelets. Transfusion. 2002;42(2):177-82. https:// doi.org/10.1046/j.1537-2995.2002.00015.x

38. Hitzler WE. [Single-donor (apheresis) platelets and pooled whole-blood-derived platelets--significance and assessment of both blood products]. Clin Lab. 2014;60(4):S1-39. Review. German.

39. Dumont $\mathrm{L}$, et al. Screening of single-donor apheresis platelets for bacterial contamination: the PASSPORT study re- sults. Transfusion. 2010;50(3):589-99. doi: 10.1111/j.15372995.2009.02460.x. Epub 2009 Nov 19.

40. Ness $P$, Braine $H$, King $K$, Barrasso $C$, Kickler T, Fuller A, et al. Single-donor platelets reduce the risk of septic platelet transfusion reactions. Transfusion. 2001 ;41(7):857-61.

41. Greening DW, et al. Preparation of Platelet Concentrates for Research and Transfusion Purposes. Methods Mol Biol. 2017, 1619:31-42. doi: 10.1007/978-1-4939-7057-53.

42. Huisman EL, de Silva SU, de Peuter MA. Economic evaluation of pooled solvent/detergent treated plasma versus single donor fresh-frozen plasma in patients receiving plasma transfusions in the United States. Transfus Apher Sci. 2014;51(1):17-24.

43. Dalal N, Klein LM. Single Donor Vs. Acrodose Platelets in Oncology Patients: A Single Institutional Experience. Biol Blood Marrow Transplant. 2015;21(2):S247-8.

44. Riga $A$, et al. Blood donors--Serious adverse reactions (SAR) 2010-2014 EFS Châteauroux, France. Transfus Clin Biol. 2015;22(2):62-5. https://doi.org/10.1016/j. tracli.2015.04.002

45. Tinmouth AT, et al. Platelet immunopathology and therapy: a Canadian Blood Services Research and Development Symposium. Transfus Med Rev. 2006;20(4):294-314. https:// doi.org/10.1016/j.tmrv.2006.05.008

46. Williamson LM, Lowe $\mathrm{S}$, Love EM, Cohen $\mathrm{H}$, Soldan $\mathrm{K}$, McClelland DB, Skacel P, Barbara JA. Serious hazards of transfusion (SHOT) initiative: analysis of the first two annual reports. BMJ. 1999;319(7201):16-9. 